
MEDIUM-ENERGY HADRON PARALLEL SESSIONS

Autrop(s): J. B. McClelland

Sunmitted to: 'The Proceedings of the IVth Conference on the Intersections between

Particle and Nuclear Physics, T'iscson, A7., May 24-29, 1991 


\title{
SUMMARY OF LOW-ENERGY ASPECTS OF QCD AND MEDIUM-ENERGY HADRON PARALLEL SESSIONS
}

\author{
John B. McClelland
}

Los Alamos National Laboratory, Los Alamos, NM 87545

Two sessions were organized dealing with low energy aspects of QCD. The first dealt with the issue of QCD dibaryons. A common underlying feature of all QCD models of dibaryons is confinement, that is a localization energy for the quarks and color-magnetic spin-splitting. While these effects usually oppose each other, there are cases in which both effects produce attraction in the six-quark state. These include the $S=0, T=0, J=3$ (" $\Delta \Delta ")$ and $S=-3, T=1 / 2, J=2$ ("N $\Omega$ ") states. The binding energies for these states vary widely among various models, but both states appear to always be bound. The quark structure of these states is not that of two baryons. Predictions of the relevant cross sections seem to indicate that such states should be experimentally observable. Plans to search for the $S=0$ state using the ${ }^{3} \mathrm{He}\left(\pi^{+}, \mathrm{p}\right) \mathrm{X}$ reaction are underway at LAMPF. These experiments would benefit greatly from higher-energy pion beams of good quality. Non-observation of these states below the predicted levels may in fact be more significant for QCD modeling than their observation, since it would point to an underlying flaw in the assumptions made in all of these models. The scope of this session included both a theoretical and experimental overview of this area of reseurch, together with more detailed talks on several searches both completed and in progress.

Evidence was presented for $S=-1, B=2$ states in the $K^{-} d \rightarrow X \pi^{-}$and $K^{-}$ ${ }^{3} \mathrm{Hle} \rightarrow \mathrm{nX} \pi^{+}$reactions at $870 \mathrm{MeV} / \mathrm{c}$ performed at $\mathrm{BNL}$ and for observation of the sequential weak decay of double hypernuclei seen in a KEK experiment. The later observation apparently places new lower limits on the mass of the H-dibaryon. An update was presented on the search for the H-dibaryon using the new BNL. 2.0 GeV/c kaon beamline.

The second session centered on mostly low-energy tests of QCD. The pion-nucleon sigma term is a measure of the chiral asymmetry generated by the $u$ and $d$ quark masses in QCD. It can be derived from the baryon mass spectrum and irom low-energy $\pi$-nucleon 
scattering data. Extrapolation to the unphysical Cheng-Dashen point provides a determination of the strange-quark content of the proton. Previous analysis had indicated that about half the proton mass was due to $s \bar{s}$ contributions. The data used in these analysis, however, appear to be inconsistent. A new analysis using only a limited data set brings the mass fraction down to less than $15 \%$ with uncertainties consistent with zero. It is estimated that roughly half the uncertainty in these calculations is due to inconsistencies in the data with an equal amount coming from theoretical uncertaintics. Several of the meson factories are gearing up for a new round of experiments to better derine the experimental quantities, including polarized target and single charge-exchange data.

Another test of chiral symmetry rciates to $\pi N \rightarrow \pi \pi N$ data near threshold. Exact chiral symmetry implies zero $\pi \pi$ scattering at zero relative momentum. Previous analysis suffered from several difficulties, both experimental and theoretical. The present global analysis includes new data which now constitute a complete set in all channels, which overdetermines the problem. Discrepancies in the data seem to have now been resolved. The two $\pi \pi$ scattering lengths derived from the data now appear to be consistent with model calculations.

Also relevant to $\pi$-nucleon scattering analysis is the isospin symmetrybreaking formalism discussed in this session. Contributions from various commonly-known isospin-violating processes were given. The mathematical properies of all the isospin-breaking operators were developed and experimental strategies for extracting the corresponding amplitudes were suggested.

Finally, two important dynamical properties of hadrons were discussed in which QCD models make firm predictions. These are the electric and magnetic polarizabilities, which characterize the induced transient dipole moments of hadrons in an external electromagnetic field. Plans to measure these quantities for $\pi, K, \Sigma$ and other hadrons using a highenergy $\pi$ beam at FNAL on a photon target via radiative pion scatiering were discussed. This technique also provides access to the radiative decaly of hadrons.

Two sessions were organized dealing primarily with medium-energy hadron reactions. The spin degree of freedom has been at the center of discussion in this area of physics for some time now. It has been 
said that every time a new spin observable is measured, something new is learned. Spin observables have been at the heart of the debate among various approaches to proton-nucleus scattering in recent years. New technology and experimental facilities continue to offer new opportunities for this important line of investigation. In particular, la.ge-volume high-power polarized nuclear targer technology has opened a new area of experimental studies. Recent results from LAMPF on pion elastic scattering and charge-exchange reactions and TRIUMF elastic scattering from polarized nuclear targets were reported. These data provide explicit spin-dependent information on the $\pi$-nucleus interaction.

Further theoretical interpretation of proton scattering results from polarized nuclear targets was also presented. Both relativistic and nonrelativistic distorted-wave calculations are now in reasonable agreement with the LAMPF $\mathrm{p}+{ }^{13} \mathrm{C}$ data. These data are sensitive to parts of the effective $N N$ interaction basically untested with unpolarized targets and therefore provide a testing ground for models of the effective interaction.

Recent developments in optically pumped polarized ${ }^{3} \mathrm{He}$ target technology at TRIUMF have produced a good figure of merit (high density and polarization) for these targets. The first set of correlation experiments using this target are now in progress. This type of target may also be useful as a polarized neutron target, in that about $90 \%$ of the target polarization is carried by the neutron. Several high-energy experiments plan to use similar targets to measure spin structure functions for the neutron.

The first measurement of a complete set of polarization transfer observables for quasifree scattering in the $(p, n)$ reaction using the new Neutron Time-of-Flight facility at LAMPF were reported. The interesting aspect of tilese measurements is that the spin-longitudinal $\left(R_{L}\right)$ and spin-transverse $\left(R_{T}\right)$ nuclear responses can be extracted from such data. Models of the particle-hole interaction predict significant enhancement in the ratio of $R_{L} / R_{T}$ as compared to frec scattering due to the attractive $\pi$ and repulsive $\rho$ interactions. These enhancements are not observed in the data at the momentum transfer measured. Other momentum transfer measurements are being planned.

Spin also plays an important role in low-energy neutron-nucleus scattering. Experiments in progress and being planned at KI:K to 
measure parity violation and time-reversal invariance in these systems were reported. Advantage is taken of the fact that the nucleus provides a large enhancement of the parity nonconserving effects due to the weak interaction, such that effects as large as $10 \%$ have been observed.

Medium-energy meson reactions provide a variety of approaches in the study of nuclear physics. Near $1 \mathrm{GeV} / \mathrm{c}$, the $\mathrm{K}^{+}$has a long mean free path in nuclei and therefore provides a weakly interacting probe with which to study nuclear-medium effects. New data were presented on $\mathrm{K}^{+}$total cross sections, extending the range up to ${ }^{40} \mathrm{Ca}$. These data are used to explore issues such as swelling or partial deconfinement of the nucleon in the nucleus or modification of the vector mesons within the medium, for instance. In general, the inclusion of these unconventional effects impruves the agreement between theory and experiment above $600 \mathrm{MeV} / \mathrm{c}$ laboratory momentum, while more conventional approaches give a better description of the data at the lower momenta. The inability of various models to reproduce the momentum dependence observed in the data must be resolved before any conclusions about medium effects can be reliably made.

Pion absorption plays a central role at intermediate energies, accounting for roughly one-third of the total cross section. Inasmuch as two or more nucleons participate, pion absorption should be sensitive to short-range effects. Large-solid-angle detectors are either in operation or being planned at all the meson facilities in order to extend these measurements. In addition, a combined effort using pions, electrons, and photons is underway to better characterize this complex process.

Studies of the nonleptonic weak decays of lambda hypernuclei were discussed. A hypernucleus can decay via mesonic and nonmesonic decay modes. These processes require an effective weak Hamiltonian which takes into account modification of the weak force due to the presence of strongly interacting particles. The nuclear decays can be used to test the $\Delta I=I / 2$ rule within the nuclear medium, for instance.

Previous results from reaction-cross-section measurements and dissociation of radioactive nuclear beams have indicated a large neutron halo in ${ }^{1} \mathrm{Li}$. The pion double-charge-exchange reaction is extremely sensitive to the two-neutron radius and can be used as an 
independent test of these results. Analysis of the neutron radius of ${ }^{11} \mathrm{Li}$ as obtained from the ${ }^{11} \mathrm{Be}\left(\pi^{-}, \pi^{+}\right)^{11} \mathrm{Li}$ reaction was reported. All ${ }^{11} \mathrm{Li}$ experiments are now consistent with a value for the neutron radius of avout $5 \mathrm{fm}$. However, the very large value derived from the dissociation measurements appears to be ruled out.

The intersection between particle and nuclear physics was especially evident in these sessions, and a bright future for new developments should be expected. We look forward to the next meeting of this conference to have lively discussions once again for the mutual benefit of these two exciting fields. 